\title{
Metal oxide gas sensors with three-parameter readout
}

\author{
Hackner, Angelika \\ EADS, Innovation Works \\ P.O.Box 81663 München
}

Conductometric metal oxide (MOX) gas sensors respond with good sensitivity to a wide range of gases. In addition such sensors feature low cost, small-size, light weight and are easy to integrate in electronic and control devices. A known drawback of MOX gas sensors is their cross sensitivity. Workarounds are gas sensor arrays consisting of several sensors with different cross sensitivity profiles. Cross sensitivity shaping can be performed by using different kinds of MOX materials, additional filter layers, different operation temperatures or by employing transient heating techniques.

In this paper, we report on MOX gas sensors which had been specifically configured to enable simultaneous readout of three different sensor signals from a single MOX layer. These single-sensor devices are able to function as a complete sensor array and thus are able to provide enhanced selectivity without necessitating multiple sensor devices.

A cross section through such a three-parameter gas sensor is shown in Fig.1. As in a conventional MOX sensor, the sensing layer is deposited on the front side of a ceramic heater substrate on top of predeposited Pt electrodes. The back surface of the substrate is fitted with a Pt meander for sensor heating and a Pt resistor for temperature control and temperature stabilization. The innovative feature in the three-parameter sensor is the collector electrode positioned at a short distance above the sensing layer, and separated by a small air gap $(\mathrm{d} \sim 1 \mathrm{~mm})$ from the sensing layer itself.

The sensor signals that can be extracted from such sensors are:

$\Delta \mathrm{T}$ : Temperature response: the thermometer function is used for sensing temperature changes due to catalytic gas interactions with the sensing layer;

$\Delta \mathrm{R}$ : Resistivity response: allows gas-induced resistance changes of the MOX layer to be monitored;

$\Delta \mathrm{l}$ : Surface ionization response: allows ion currents to be detected which derive from the thermal ionization of analyte molecules adsorbed on the MOX sensing layer.

Each single measurement principle is sensitive to the surface temperature and to the sensing material actually employed. The sensor architecture in Fig. 1 therefore provides a huge degree of flexibility for adaptation to specific measurement applications. 


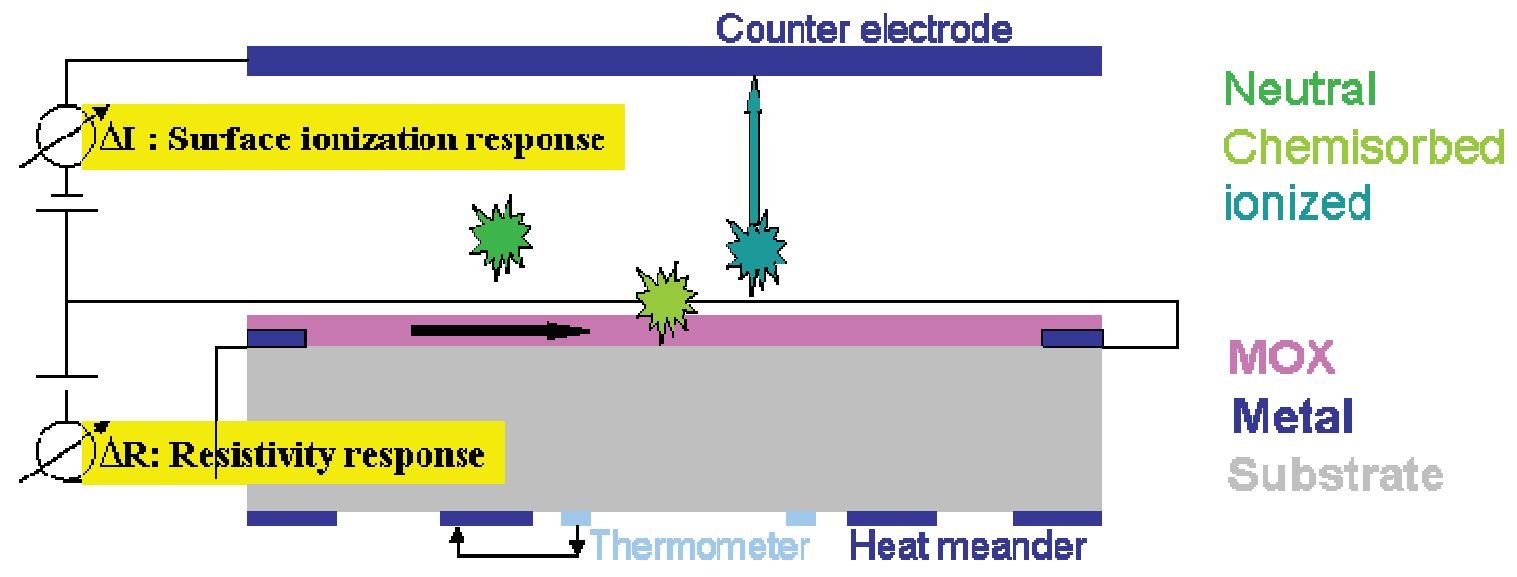

\section{$\Delta T$ : Temperature response}

Pre-set and stabilization of substrate temperature via readjustment of heating voltage

Figure 1: Sensor architecture used to study the surface ionization $(\Delta \mathrm{l})$, the resistive $(\Delta R)$ and the temperature $(\Delta T)$ response of MOX sensing layers.

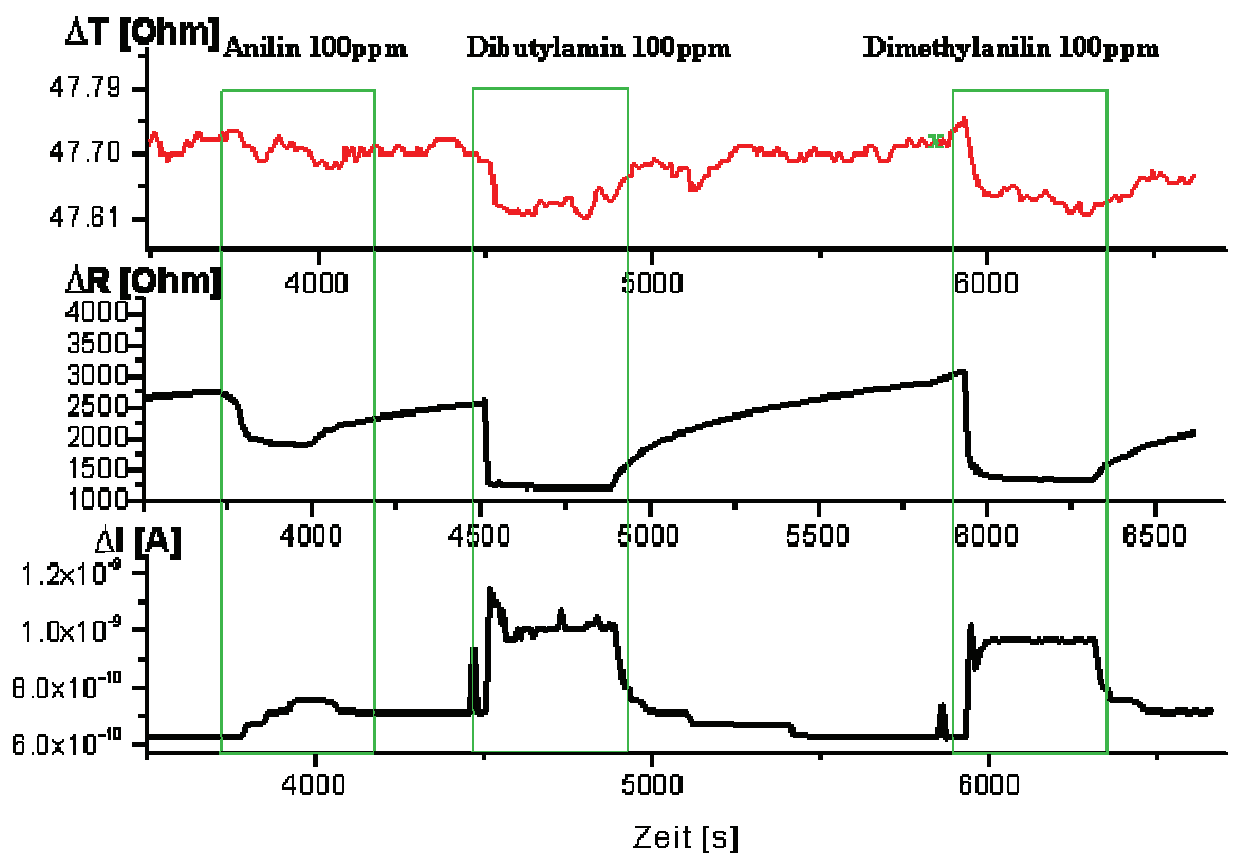

Figure 2: Result of a three-parameter detection experiment performed on a single SnO2 layer: Temperature response $(\Delta T)$, Resistivity response $(\Delta R)$ and surface ionization response $(\Delta \mathrm{l})$. 